\title{
Lambda-carrageenan treatment exacerbates the severity of cerebral malaria caused by Plasmodium berghei ANKA in BALB/c mice
}

Frances C Recuenco ${ }^{1,2,4}$, Ryo Takano², Shiori Chiba3 ${ }^{3}$, Tatsuki Sugi 1,2, Hitoshi Takemae ${ }^{2}$, Fumi Murakoshi ${ }^{1,2}$, Akiko Ishiwa ${ }^{1,2}$, Atsuko Inomata ${ }^{1}$, Taisuke Horimoto ${ }^{1}$, Yoshiyasu Kobayashi ${ }^{3}$, Noriyuki Horiuchi ${ }^{3}$ and Kentaro Kato ${ }^{1,2^{*}}$

\begin{abstract}
Background: There is an urgent need to develop and test novel compounds against malaria infection. Carrageenans, sulphated polysaccharides derived from seaweeds, have been previously shown to inhibit Plasmodium falciparum in vitro. However, they are inflammatory and alter the permeability of the blood-brain barrier, raising concerns that their use as a treatment for malaria could lead to cerebral malaria (CM), a severe complication of the disease. In this work, the authors look into the effects of the administration of $\lambda$-carrageenan to the development and severity of CM in BALB/c mice, a relatively non-susceptible model, during infection with the ANKA strain of Plasmodium berghei.
\end{abstract}

Methods: Five-week-old female BALB/c mice were infected with $P$. berghei intraperitoneally. One group was treated with $\lambda$-carrageenan (PbCGN) following the 4-day suppressive test protocol, whereas the other group was not treated $(\mathrm{PbN})$. Another group of healthy BALB/c mice was similarly given $\lambda$-carrageenan (CGN) for comparison. The following parameters were assessed: parasitaemia, clinical signs of CM, and mortality. Brain and other vital organs were collected and examined for gross and histopathological lesions. Evans blue dye assays were employed to assess blood-brain barrier integrity.

Results: Plasmodium berghei ANKA-infected BALB/C mice treated with $\lambda$-carrageenan died earlier than those that received no treatment. Histopathological examination revealed that intracerebral haemorrhages related to $C M$ were present in both groups of infected BALB/c mice, but were more numerous in those treated with $\lambda$-carrageenan than in mock-treated animals. Inflammatory lesions were also observed only in the $\lambda$-carrageenan-treated mice. These observations are consistent with the clinical signs associated with CM, such as head tilt, convulsions, and coma, which were observed only in this group, and may account for the earlier death of the mice.

Conclusion: The results of this study indicate that the administration of $\lambda$-carrageenan exacerbates the severe brain lesions and clinical signs associated with CM in BALB/c mice infected with $P$. berghei ANKA.

Keywords: Cerebral malaria, $\lambda$-carrageenan, BALB/c mouse, Intracerebral haemorrhage, Plasmodium berghei ANKA

\footnotetext{
* Correspondence: kkato@obihiro.ac.jp

'Department of Veterinary Microbiology, Graduate School of Agricultural and

Life Sciences, The University of Tokyo, 1-1-1 Yayoi, Bunkyo-ku, Tokyo

113-8657, Japan

${ }^{2}$ National Research Center for Protozoan Diseases, Obihiro University of

Agriculture and Veterinary Medicine, Obihiro 080-8555, Hokkaido, Japan

Full list of author information is available at the end of the article
} 


\section{Background}

Carrageenans are linear sulphated polysaccharides derived from seaweeds. The three types of carrageenans are characterized according to their levels of sulphation and are identified by Greek letters: $\mathrm{K}$-carrageenan, I-carrageenan, and $\lambda$-carrageenan. $k$-carrageenan has one sulphate group and forms rigid gels, $\mathrm{t}$-carrageenan has two sulphate groups and forms soft gels, and $\lambda$-carrageenan has three sulphate groups and does not gel in solution.

Carrageenans have known anti-malarial activity in vitro and in vivo. Adams et al. previously demonstrated that carrageenans inhibit the growth and invasion of Plasmodium falciparum $3 \mathrm{D} 7$ and $\mathrm{Dd} 2$ in vitro with $\lambda$-carrageenan having the highest inhibitory activity, followed by I-carrageenan, and $\mathrm{k}$-carrageenan [1]. Further, James et al. reported that calcium carrageenan pre-treatment of $\mathrm{A} / \mathrm{J}$ mice decreased the parasitaemia and delayed death from infection with the NK65 strain of Plasmodium berghei [2].

Carrageenans are common additives in food and personal hygiene products such as toothpastes and personal lubricants. However, the use of carrageenans as additives in foods such as meat and milk has become controversial. Several studies have linked carrageenans to inflammatory bowel disease [3] and to the development of several allergies $[4,5]$ and even mammary carcinoma [6]. Carrageenans have been used to induce inflammation in inflammatory models. Carrageenan-induced inflammation in the paw of mice or rats is a classic model of oedema formation and hyperalgesia that is useful in the development of non-steroidal anti-inflammatory drugs. Among the three types of carrageenan, $\mathrm{k}$-carrageenan was found to be the most inflammatory in the paw oedema model, whereas $\lambda$-carrageenan had the highest anticoagulant activity in vitro [7].

Carrageenan-induced inflammation involves the release of histamine and serotonin followed by the release of prostaglandins, protease, and lysosomes, producing oedema [8]. Huber et al. showed that 72-h exposure of rats to $\lambda$-carrageenan alters the permeability of the blood-brain barrier [9]. Given that dysfunction of the blood-brain barrier has been specifically observed in cerebral malaria, carrageenans could cause this serious side effect if used as anti-malarial drugs in clinical practice.

Human cerebral malaria (HCM) is the most serious and often fatal complication of severe P. falciparum malaria. Patients inflicted with HCM become comatose and show neurological symptoms, including seizures, retinopathy, brainstem alterations, and brain swelling. Those who survive $\mathrm{HCM}$ develop long-term neurological sequelae, as well as cognition and behavioural deficits [10].

Although the pathogenesis of HCM is still poorly defined, the main cause is thought to involve the sequestration of infected red blood cells (iRBC) in brain capillaries
[11]. Leukocytes and platelets get sequestered on the endothelium, which leads to occlusion of brain capillaries and poor microvascular flow, decreased nutrient supply to the brain, and damage to vascular walls that ultimately leads to haemorrhages and neuronal alterations [12]. Sequestration is a characteristic of $P$. falciparum malaria that involves molecular adhesion between parasite proteins, such as PfEMP1, the knob protein expressed on the surface of infected erythrocytes, and ligands on endothelial cells, such as CD36, thrombospondin, and ICAM-1 [13].

Experimental cerebral malaria (ECM) can be demonstrated by using the rodent malaria model $P$. berghei ANKA in susceptible mouse strains, such as C57BL/6 mice. However, the use of this ECM mouse model highly controversial because not all aspects of HCM can be reproduced. The most accepted mechanism underlying the development of ECM involves the accumulation of inflammatory cells in the brain, a characteristic of encephalitis [14], rather than sequestration of infected red blood cells.

Here, the authors investigate whether administration of $\lambda$-carrageenan can contribute to the development of cerebral malaria in $\mathrm{BALB} / \mathrm{c}$ mice, a relatively non-susceptible model of experimental $\mathrm{CM}$ during infection with the ANKA strain of $P$. berghei. Histopathological analyses were also performed and the severity of the brain lesions in infected-mice treated with $\lambda$-carrageenan was evaluated.

\section{Methods}

\section{Ethics statement}

The mice used in the study were maintained in the animal care facility of NRCPD, Obihiro University of Agriculture and Veterinary Medicine under controlled conditions and were given commercial feeds and water ad libitum. The protocol for the experiments was approved by the Committee on the Animal Experiments of the Obihiro University of Agriculture and Veterinary Medicine (Approval number: 25-153).

\section{Parasites and animals}

Frozen stock of $P$. berghei ANKA parasites were provided by Dr. Motomi Torii (Ehime University), and were passaged in BALB/c mice. Female BALB/c mice, five weeks of age, were purchased from CLEA Japan (Tokyo, Japan) and used in the study.

\section{Infection and treatment}

Donor mice were infected intraperitoneally with $10^{6}$ parasitized red blood cells from a cryopreserved stock. When the parasitaemia reached around 15\%, infected blood was collected by cardiac puncture and mixed with PBS. Two groups of experimental mice were infected intraperitoneally with $10^{7}$ parasitized red blood cells: the first group was the untreated group ( $\mathrm{PbN}$ group) and 
the other group was the carrageenan-treated group (PbCGN group). Another group of uninfected mice was also treated with $\lambda$-carrageenan (CGN group). $\lambda$ carrageenan (SIGMA) was dissolved in PBS $(2 \mathrm{mg} / \mathrm{ml})$ at $65^{\circ} \mathrm{C}$ and passed through a $0.45-\mu \mathrm{m}$ filter (Millipore) for sterilization prior to use. $\lambda$-carrageenan was given intraperitoneally at a dose of $25 \mathrm{mg} / \mathrm{kg}$ BW following the four-day suppressive test. In this protocol, the infection day is designated as day 0 . The first treatment is given $2 \mathrm{~h}$ after infection, and then subsequent treatments are given every $24 \mathrm{~h}$ until day 3 post-infection, for a total of four treatments.

To determine the survival rate, half of the infected mice in each group were observed for clinical signs of cerebral malaria until the mice eventually succumbed to the disease. The other half of each group was sacrificed under terminal isoflurane anesthesia at the presumed onset of cerebral malaria for histopathological examination and Evans blue dye assays.

\section{Parasitaemia, ECM signs, and survival monitoring}

Parasitaemia levels of both groups of infected mice $(n=4)$ were monitored from days 4 to 10 post-infection by using Giemsa-stained thin blood smears. Ten oil immersion objective fields were examined each with 200-300 red blood cells. The infected mice were observed for general signs of malaria such as ruffled fur, and hunching posture, and for ECM-related signs such as wobbly gait, head tilt, limb paralysis, convulsions, and coma. Other behavioural parameters monitored included reactions to stimuli such as exploration of a new environment and touch reflexes $[15,16]$. Deaths were promptly recorded.

\section{Histopathology, intracerebral haemorrhage scoring, and statistical analysis}

The infected mice were sacrificed under terminal isoflurane anesthesia once the clinical signs of ECM were observed in some or all members of the treatment groups. This corresponded to day 5 p.i. in all members of the $\mathrm{PbCGN}$ group $(\mathrm{n}=4)$. Although no signs of CM were observed, the $\mathrm{PbN}$ mice $(\mathrm{n}=4)$ were also sacrificed at this time. Normal and healthy mice $(\mathrm{N}, \mathrm{n}=4)$ and uninfected, carrageenan-treated mice $(\mathrm{CGN}, \mathrm{n}=4)$ were also sacrificed at the same time for comparison. Brains as well as other vital organs including kidneys, livers, hearts, lungs, and spleens were collected and fixed in $4 \%$ paraformaldehyde and embedded in paraffin. The organs were sectioned at $5 \mu \mathrm{m}$ and were stained with haematoxylin-eosin (HE) stain. The brain sections were examined for haemorrhages and inflammation. Intracerebral haemorrhages were recorded [16]. Statistically significant differences in the number of intracerebral haemorrhages were determined by using the Mann-Whitney U test. Values were considered to be significantly different when the $P$ value was less than 0.05 . The organ sections were examined for any significant lesions.

\section{Assessment of vascular leakage at the blood-brain barrier by use of Evans blue dye perfusion}

On day 5 post-infection, $200 \mu \mathrm{l}$ of $1 \%$ Evans blue dye was injected into the tail veins of representative mice $(n=3-4)$ from each treatment group. After $1 \mathrm{~h}$, the mice were sacrificed and their brains were collected and examined for bluish discoloration. The brains were then placed in $4 \%$ paraformaldehyde for $48 \mathrm{~h}$ to extract the Evans blue dye, and the absorbance was measured at a wavelength of $600 \mathrm{~nm}$ [15]. For this, the results of two independent assays were pooled. Absorbance readings were carried out in triplicates and the mean of three replicates was computed. Statistically significant differences in the absorbance of Evans blue dye between the groups were determined by using Tukey's multiple comparison test. Values were considered to be significantly different when the $P$ value was less than 0.05 .

\section{Results}

\section{Effects of $\lambda$-carrageenan treatment on parasitaemia and} course of infection

Lambda-carrageenan poorly inhibited the growth of $P$. berghei ANKA, as shown by the hyperparasitaemia in the infected mice (Figure 1A), and did not improve the survival of the infected mice (Figure 1B). These findings differ from the report by James and Alger [2], who found that A/J Swiss mice infected with $P$. berghei NK65 survived for up to 28 days when pre-treated with calcium carrageenan intraperitoneally, and from the finding of Adams that $\lambda$ carrageenan effectively inhibited the growth and invasion of red blood cells by $P$. falciparum in in vitro experiments [1].

In the experiments monitoring parasitaemia, ECM, and survival, all $P$. berghei ANKA-infected mice exhibited signs of ruffled fur, hunching, and decreased reaction to stimuli on day 5 post-infection. PbCGN mice showed limb paralysis, convulsions, head tilt, and coma and died soon afterwards. On the same day, $\mathrm{PbN}$ mice showed none of these neurological signs. Deaths in the $\mathrm{PbN}$ mice group were first seen on day 7 p.i and all of the $\mathrm{PbN}$ mice died by day 13 p.i. Signs observed in the $\mathrm{PbN}$ mice that started to die from day 7 until day 13 p.i. included general weakness and lethargy, while signs that are related to ECM, specifically, limb paralysis, convulsions, and head tilt were not seen.

\section{Gross pathology and histopathology of the brains of Plasmodium berghei-infected BALB/c mice}

Haemorrhages were visible on the brains of the PbCGN mice that showed clinical signs of $\mathrm{CM}$ and were sacrificed on day 5 p.i. $(n=4)$ (Figure 2A). Similarly, haemorrhages were also observed in the $\mathrm{PbN}$ mice that were sacrificed 

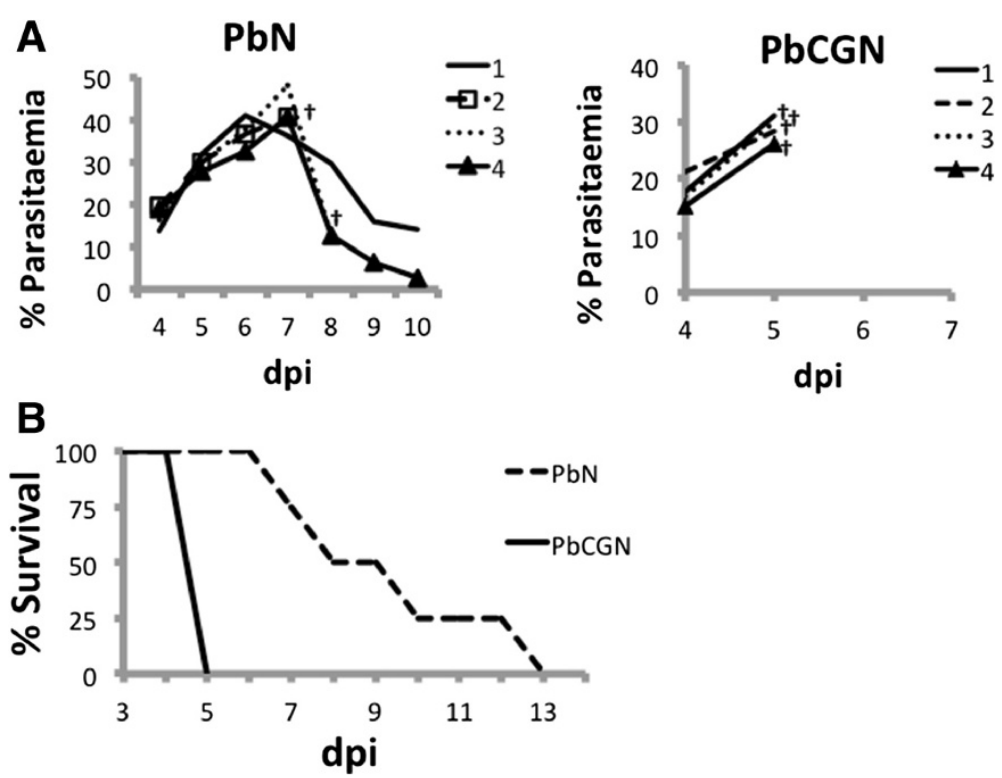

Figure 1 Parasitaemia and survival profiles of $P$. berghei ANKA-infected BALB/c mice. (A) Four mice per group were infected with $P$. berghei ANKA, and were not treated $(\mathrm{PbN})$ or treated with $\lambda$-carrageenan $(\mathrm{PbCGN})$. The parasitaemia in the blood microvessels is shown for each animal per group $(n=4)$. $\lambda$-carrageenan treatment $(25 \mathrm{mg} / \mathrm{kg})$ did not inhibit the growth of $P$. berghei ANKA in vivo using the four-day suppressive test. The dagger ( + ) indicates mouse death. (B) Survival profiles of $P$. berghei ANKA-infected BALB/C mice: $\lambda$-carrageenan-treated (PbCGN); not treated $(\mathrm{PbN})$.

on the same day $(\mathrm{n}=4)$ (Figure $2 \mathrm{~B})$. These $\mathrm{PbN}$ mice, however, did not necessarily show neurological signs related to cerebral malaria. This result shows that in the $\mathrm{BALB} / \mathrm{c}$ mouse, which is considered to be resistant to the development of CM by $P$. berghei ANKA infection, in the absence of clear neurological signs, haemorrhagic brain lesions can be observed.

To further characterize the lesions in the brains of mice infected with $P$. berghei ANKA and then treated with $\lambda$ carrageenan, two independent experiments for histopathological examinations were done with similar observations. No apparent lesions were observed in the brain sections of uninfected, healthy carrageenan-treated mice (CGN group,

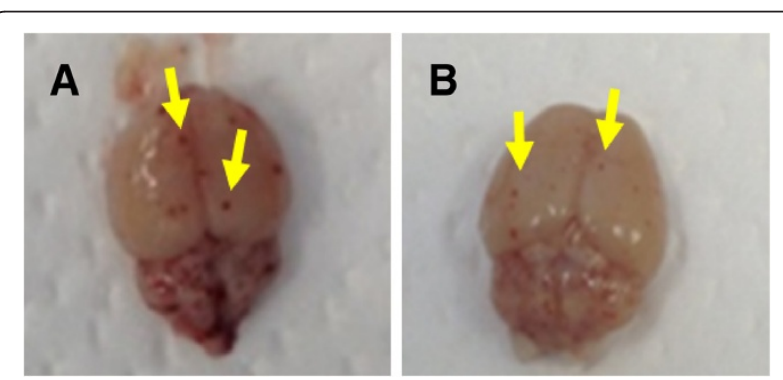

Figure 2 Gross lesions on the brains of infected mice. (A) From $\mathrm{PbCGN}$ mice that were sacrificed at the presumed onset of cerebral malaria 5 days p.i. showing clinical signs such as head tilting and convulsions. (B) From a Pb-infected, untreated (PbN) mouse sacrificed on day 5 p.i. that did not show any signs associated with $\mathrm{CM}$. Yellow arrows show petechial haemorrhages.
Figure 3A). By contrast, microthrombi, intracerebral haemorrhages, and the presence of iRBCs in brain vessels were observed in both PbN (as shown in Figure 3B) and PbCGN (Figure $3 \mathrm{C}$ and D) animals. Perivascular infiltrations of inflammatory cells around the microthrombi were also observed in the PbCGN group and not as much in the PbN group. In addition, hyperplastic endothelium of brain blood vessels was observed only in the PbCGN group (Figure $3 \mathrm{C}$ and D). These results indicate that the administration of $\lambda$ carrageenan to BALB/c mice infected with $P$. berghei ANKA caused more severe histopathological features associated with cerebral malaria.

Histopathology of other vital organs revealed malaria pigment deposits in lung sections and in the Kupffer cells in the livers of both the PbN and PbCGN groups. The presence of necrotized lymphocytes and follicular hyperplasia was also observed in the spleens of both infected groups. No apparent lesions were observed in the kidneys or hearts of these infected mice. These results show that $\lambda$-carrageenan treatment had no atypical effects on the other vital organs of the $P$. berghei-infected $\mathrm{BALB} / \mathrm{c}$ mice.

\section{Intracerebral haemorrhage counts}

Intracerebral haemorrhages observed in sections from four major regions of the brain, namely, the frontal lobe, diencephalon, occipital lobe, and cerebellum, were counted and tallied $(\mathrm{n}=4, \mathrm{PbN}$ and PbCGN, respectively). There were no statistically significant differences 


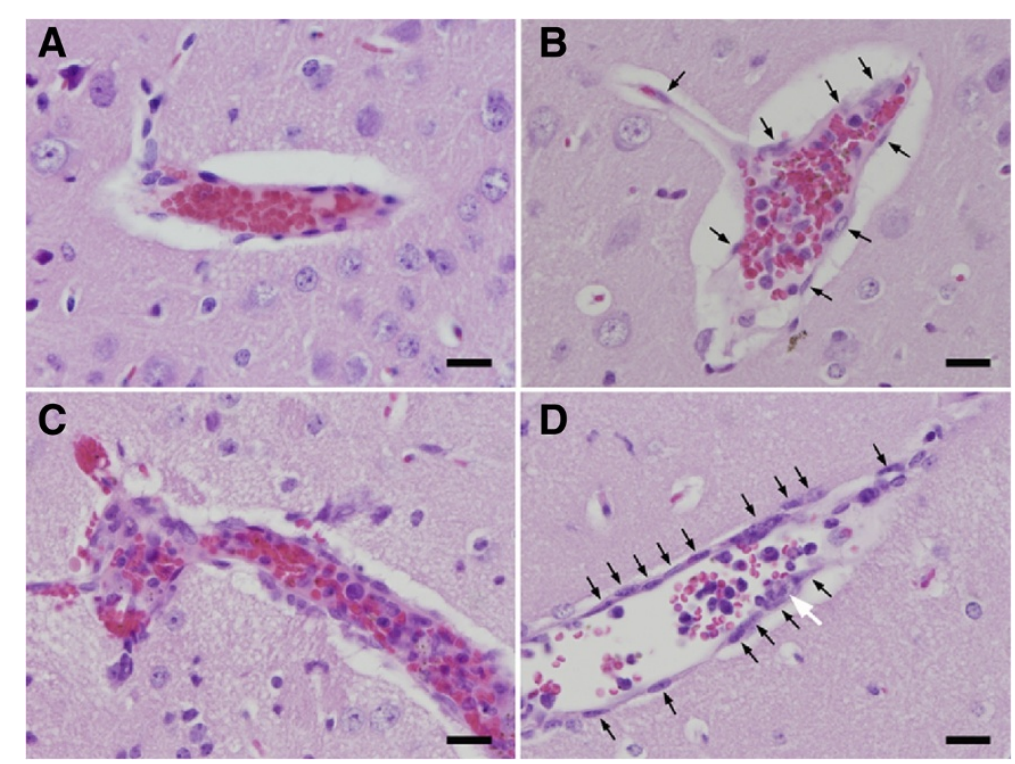

Figure 3 Brain histopathology. (A) CGN group. No apparent lesions are observed (Haematoxylin-Eosin stain). Bar, 20 um. (B) PbN group. Microthrombus consisting of fibrin pigmented RBCs and accumulated mononuclear cells, is observed. Perivascular infiltration of inflammatory cells is not observed. Vascular endothelium is not hyperplastic (arrows indicate endothelial cells). Bar, 20 mm. (C) PbCGN group. Microthrombus consisting of fibrin, pigmented RBCs, pigmented macrophages, and mononuclear cells is observed. Perivascular infiltration of inflammatory cells, haemorrhage in perivascular space, and hyperplastic vascular endothelium are also observed. Bar, 20 m. (D) PbCGN group. Infected RBCs and inflammatory cells can be seen within the blood vessels of the cerebrum. Note the hyperplastic endothelium, characterized by increased number of endothelial cells (arrow). Adhesive Pb-laden macrophages (shown by white arrow) can also be seen. Bar, $20 \mu \mathrm{m}$.

in the number of intracerebral haemorrhages between the $\mathrm{PbN}$ and PbCGN groups. However, haemorrhages were observed in all regions of the brains of all of the PbCGN mice, whereas they were observed in only half of the $\mathrm{PbN}$ mice tested (Table 1). These findings are consistent with the increased severity of the pathology in the PbCGN mice relative to the $\mathrm{PbN}$ mice.

\section{Assessment of vascular leakage at the blood-brain barrier by using Evans blue dye perfusion}

To further evaluate the effects of $\lambda$-carrageenan on the integrity of blood-brain barrier (BBB), and its contribution to the early death of the mice infected with $P$. berghei ANKA, the permeability of the blood-brain barrier was assessed by using Evans blue assay. Representative

Table 1 Intracerebral haemorrhage counts

\begin{tabular}{|c|c|c|c|c|c|c|c|c|c|}
\hline \multirow{2}{*}{$\begin{array}{l}\text { Haemorrhage } \\
\text { score }\end{array}$} & \multicolumn{4}{|c|}{$\mathrm{PbN}$} & \multicolumn{4}{|c|}{ PbCGN } & \multirow{2}{*}{$\begin{array}{l}\text { Mann-Whitney } \\
P\end{array}$} \\
\hline & $\bar{A}$ & B & $C$ & D & $\bar{E}$ & $F$ & G & $\mathrm{H}$ & \\
\hline ontal Lobe & 2 & 0 & 0 & 4 & 6 & 4 & 9 & 3 & 0.05755 \\
\hline Diencephalon & 5 & 0 & 0 & 1 & 6 & 4 & 9 & 3 & 0.1059 \\
\hline Occipital Lobe & 4 & 2 & 0 & 6 & 2 & 4 & 7 & 2 & 0.6552 \\
\hline 年 & 5 & 0 & 0 & 1 & 4 & 6 & 4 & 4 & 0.1367 \\
\hline Total & 16 & 2 & 0 & 12 & 14 & 19 & 27 & 11 & 0.1489 \\
\hline
\end{tabular}

Statistically significant differences in intracerebral haemorrhage counts were determined by using the Mann-Whitney test. Values were considered to be significantly different when the $P$ value was less than 0.05 . images of the brains of mice from each group are shown in Figure 4. The brains of the PbN and PbCGN mice (Figure $4 \mathrm{C}$ and $\mathrm{D}$, respectively) absorbed the dye, indicating increased permeability of the blood-brain barrier compared with that of the brains of normal mice and CGN mice (Figure 4A and B, respectively). As expected, CGN mice showed increased levels of blood-brain barrier leakage relative to the levels in the brains of normal mice. However, there were no statistically significant differences in the levels of Evans blue dye leakage from the mouse brains between the $\mathrm{PbN}$ and PbCGN groups (Figure 4E). These results indicate that not only the altered integrity of blood-brain barrier but also other factors may lead to this severe complication of malaria in mice infected with $P$. berghei ANKA.

\section{Discussion}

Here, the in vivo efficacy and safety of $\lambda$-carrageenan as an anti-malarial drug was evaluated. The results show that $\lambda$ carrageenan poorly inhibited the growth of $P$. berghei ANKA in BALB/c mice and caused more severe brain lesions, leading to the early death of the mice. BALB/c mice, which are considered to be relatively resistant to the consequences of cerebral malaria caused by the ANKA strain of $P$. berghei [10,17-19], were chosen as model to assess the effects of the administration of $\lambda$-carrageenan to the development of clinical signs associated with cerebral malaria. Both groups were shown to develop a high parasitaemia, but the 

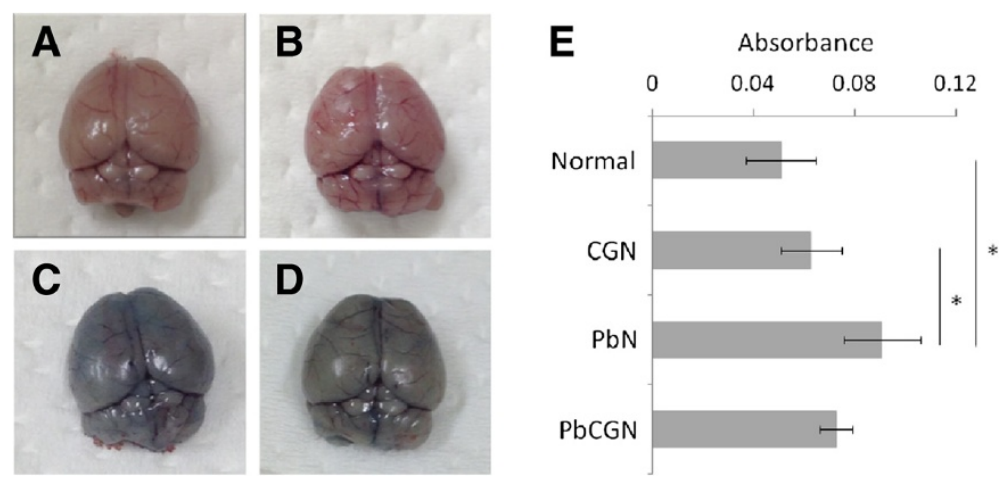

Figure 4 Assessment of vascular leakage at the blood-brain barrier by use of Evans blue dye perfusion. Two hundred microliters of 1\% Evans blue dye was injected into the tail veins of each mice on day 5 post-infection. After $1 \mathrm{~h}$, the mice were sacrificed using terminal isoflurane anesthesia and the brains were collected. Representative brains are shown: (A) Normal healthy mouse (N), (B) Carrageenan-treated mouse (CGN), (C) P. berghei ANKA-infected, untreated mouse (PbN), (D) P. berghei ANKA-infected, carrageenan-treated mouse (PbCGN). (E) The mean absorbances at $600 \mathrm{~nm}$ wavelength and standard deviations of extracted dye after placing the brains in $4 \%$ paraformaldehyde for $48 \mathrm{~h}$ are shown for each group. Statistically significant differences in mean absorbances were determined by using Tukey's multiple comparison test (*: $P$ value $<0.05$, ns: not significant).

infected BALB/c mice treated with $\lambda$-carrageenan showed more signs related to ECM and died earlier than the untreated mice. The attempts to show that $\lambda$ carrageenan treatment could cause more severe cerebral malaria through histopathology and Evans blue dye assays revealed almost the same results for both groups of infected mice. The levels of blood-brain barrier leakage were similar between the $P$. berghei-infected mice that were mock-treated and those that were administered $\lambda$-carrageenan; and the histopathological findings on the brains show the presence of intracranial haemorrhages for both groups. It is important to note that with the small sample size used in this study, the statistical data presented here should be interpreted with caution [20-22].

It is also possible that although the clinical signs associated with ECM in the untreated group were not observed, these mice may also have developed ECM, as reported by Neill and Hunt [23]. It will be interesting to explore the underlying mechanisms as to why clear neurological signs were not as evident in the untreated group given that there was hyperparasitaemia, leakage in the $\mathrm{BBB}$ and presence of intracranial haemorrhages. These results indicate that there may be other factors involved in the early death of mice treated with $\lambda$ carrageenan during $P$. berghei ANKA infection. One can also consider the possibility that the hyperplastic endothelium of the brain blood vessels compensated for the blood-brain barrier leakage in the PbCGN group.

Lambda-carrageenan is known to induce inflammatory pain and to alter the permeability of the blood-brain barrier in several animal models [9,24]. Moreover, administration of $\lambda$-carrageenan increases the expression of ICAM-1, which plays a key role in the sequestration of iRBCs in cerebral malaria under experimental and natural conditions $[25,26]$.

By contrast, $\lambda$-carrageenan is known to activate the innate immunity that depends on Toll-like receptor 4 (TLR4) and Myd88 [5]. Given that the activation of TLR4/ Myd88 signaling causes acute inflammatory injury [27], one could argue that the early deaths of mice treated with $\lambda$-carrageenan during the $P$. berghei ANKA infection could be attributed to the dysregulation of innate immunity rather than the dysfunction of the blood-brain barrier. Further studies are warranted to elucidate the molecular basis of this exacerbation of pathology.

Malaria remains a threat to human health and there is an urgent need to develop and test novel compounds. The results presented here suggest that $\lambda$-carrageenan, a sulphated polysaccharide which is a common food additive and ingredient in household products and has been found to inhibit the invasion of red blood cells by malaria merozoites in vitro, may be unsuitable for the treatment of clinical malaria.

However, other sulphated polysaccharides, including heparin, fucoidan, and dextran sulfate [28,29] have shown promising in vitro anti-malarial activity. An example is the previous report on gellan sulfate [30] that demonstrated that chemical modification, namely, sulphation, could change the characteristics of these compounds. Given that these polysaccharides are still considered promising potential anti-malaria drugs, chemical modification might decrease the side effects of such compounds.

\section{Conclusion}

This study shows the potential toxicity of $\lambda$-carrageenan as an antimalarial using the $\mathrm{BALB} / \mathrm{c}$ mice as model of 
ECM. We find the usefulness of this rodent model in elucidating CM pathogenesis and evaluating promising antimalarial candidates in vivo and more importantly the safety profile of anti-malarial compounds that could not be envisaged if only in vitro experiments were conducted.

\section{Abbreviations}

CM: Cerebral malaria; ECM: Experimental cerebral malaria; HCM: Human cerebral malaria; BBB: Blood-brain barrier; iRBCs: Infected red blood cells; H-E: Haematoxylin-eosin stain.

\section{Competing interests}

The authors declare that they have no competing interests.

\section{Authors' contributions}

FCR and KK designed the study. FCR performed all of the animal experiments and wrote the manuscript. RT conducted the statistical studies and co-wrote the manuscript. SC, NH, and YK prepared the histopathology slides and performed all of the histopathological examinations. TS, HT, FM, A. Ishiwa, A. Inomata, and TH contributed to the data analysis. KK edited the manuscript and supervised the study. All authors have read and approved the final version of the manuscript.

\section{Acknowledgements}

This study was supported by Grants-in-Aids for Young Scientists, and Scientific Research on Innovative Areas (3308) from the Ministry of Education, Culture, Science, Sports, and Technology (MEXT) and for Research on global health issues from the Ministry of Health, Labour, and Welfare of Japan, by Bio-oriented Technology Research Advancement Institution (BRAIN), by the Naito Foundation, and by the Programme to Disseminate Tenure Tracking System from the Japan Science and Technology Agency (JST).

We would also like to thank Dr. Maria Shirley Herbas of the NRCPD, Obihiro University of Agriculture and Veterinary Medicine for providing training on tissue handling and collection.

\section{Author details}

'Department of Veterinary Microbiology, Graduate School of Agricultural and Life Sciences, The University of Tokyo, 1-1-1 Yayoi, Bunkyo-ku, Tokyo 113-8657, Japan. ${ }^{2}$ National Research Center for Protozoan Diseases, Obihiro University of Agriculture and Veterinary Medicine, Obihiro 080-8555, Hokkaido, Japan. ${ }^{3}$ Department of Basic Veterinary Medicine, Obihiro University of Agriculture and Veterinary Medicine, Obihiro 080-8555, Hokkaido, Japan. ${ }^{4}$ Department of Veterinary Paraclinical Sciences, College of Veterinary Medicine, University of the Philippines Los Baños, Laguna 4031, Philippines.

Received: 1 September 2014 Accepted: 7 December 2014 Published: 11 December 2014

\section{References}

1. Adams Y, Smith SL, Schwartz-Albiez R, Andrews KT: Carrageenans inhibit the in vitro growth of Plasmodium falciparum and cytoadhesion to CD36. Parasitol Res 2005, 97:290-294.

2. James MA, Alger NE: Plasmodium berghei: effect of carrageenan on the course of infection in the A/J mouse. Int J Parasitol 1981, 11:217-220.

3. Moyana TN, Lalonde JM: Carrageenan-induced intestinal injury in the rat -a model for inflammatory bowel disease. Ann Clin Lab Sci 1990, 20:420-426.

4. Merlo S, Dolovich J, Listgarten C: Anaphylaxis to carrageenan: a pseudolatex allergy. J Allergy Clin Immunol 1995, 95:933-936.

5. Tsuji RF, Hoshino K, Noro Y, Tsuji NM, Kurokawa T, Masuda T, Akira S, Nowak B: Suppression of allergic reaction by $\lambda$-carrageenan: toll-like receptor $4 /$ MyD88-dependent and -independent modulation of immunity. Clin Exp Allergy 2003, 33:249-258.

6. Tobacman JK, Wallace RB, Zimmerman MB: Consumption of carrageenan and other water-soluble polymers used as food additives and incidence of mammary carcinoma. Med Hypotheses 2001, 56:589-598.

7. Silva FRF, Dore CMPG, Marques $C T$, Nascimento MS, Benevides NMB, Rocha HAO, Chavante SF, Leite EL: Anticoagulant activity, paw edema and pleurisy induced carrageenan: action of major types of commercial carrageenans. Carbohydr Polym 2010, 79:26-33.

8. Suralkar AA, Sarda PS, Ghaisas MM, Thakare VN, Deshpande AD: In-vivo animal models for evaluation of anti-inflammatory activity. Pharmainfonet 2008, 6: http://www.pharmainfo.net/reviews/vivo-animal-models-evaluationanti-inflammatory-activity. Accessed July 8, 2014.

9. Huber JD, Hau VS, Borg L, Campos CR, Egleton RD, Davi TP: Blood-brain barrier tight junctions are altered during a 72-h exposure to $\lambda$ carrageenan-induced inflammatory pain. Am J Physiol Heart Circ Physiol 2002, 283:H1531-H1537.

10. Rénia L, Howland SW, Claser C, Charlotte Gruner A, Suwanarusk R, Hui Teo T, Russel B, Ng LF: Cerebral malaria: mysteries at the blood-brain barrier. Virulence 2012, 3:193-201.

11. Aikawa M, Iseki M, Barnwell JW, Taylor D, Oo MM, Howard RJ: The pathology of cerebral malaria. Am J Trop Med Hyg 1990, 43:30-37.

12. van der Heyde HC, Nolan J, Combes V, Gramaglia I, Grau GE: A unified hypothesis for the genesis of cerebral malaria: sequestration, inflammation and hemostasis leading to microcirculatory dysfunction. Trends Parasitol 2006, 22:503-508.

13. Ponsford MJ, Medana IM, Prapansilp P, Hien TT, Lee SJ, Dondorp AM Esiri MM, Day NP, White NJ, Turner GD: Sequestration and microvascular congestion are associated with coma in human cerebral malaria. J Infect Dis 2012, 205:663-671.

14. Jennings VM, Actor JK, Lal AA, Hunter RL: Cytokine profile suggesting that murine cerebral malaria is an encephalitis. Infect Immun 1997, 65:4883-4887

15. Bopp SER, Rodrigo E, Gonzalez-Paez GE, Frazer M, Barnes SW, Valim C, Watson J, Walker JR, Schemdt C, Winzeler EA: Identification of the Plasmodium berghei resistance locus 9 linked to survival on chromosome 9. Malar J 2013, 12:316.

16. Carroll RW, Wainwright MS, Kim KY, Kidambi T, Gomez ND, Taylor T, Haldar $\mathrm{K}$ : A rapid murine coma and behavior scale for quantitative assessment of murine cerebral malaria. PLoS One 2010, 5:e13124.

17. Nacer A, Movila A, Baer K, Mikolajczak SA, Kappe SHI, Frevert U: Neuroimmunological blood brain barrier opening in experimental cerebral malaria. PLoS Pathog 2012, 8:e1002982. doi:10.1371/journal. ppat.1002982.

18. Schmidt KE, Schumak B, Specht S, Dubben B, Limmer A, Hoerauf A: Induction of pro-inflammatory mediators in Plasmodium berghei infected $\mathrm{BALB} / \mathrm{c}$ mice breaks blood-brain-barrier and leads to cerebral malaria in an IL-12 dependent manner. Microb Infect 2011, 13:828-836.

19. Taylor-Robinson AW: Validity of modelling cerebral malaria in mice: argument and counter argument. J Neuroparasitol 2010, 1:N100601. doi:10.4303/jnp/N100601. Accessed July 8, 2014.

20. Bacchetti P, Deeks SG, McCune JM: Breaking free of sample size dogma to perform innovative translational research. Sci Transl Med 2011, 3:87ps24.

21. Liu PT: Extremely small sample size in some toxicity studies: an example from the rabbit eye irritation test. Regul Toxicol Pharmacol 2001, 33:187-191.

22. De Winter JCF: Using the student's t-test with extremely small sample sizes. PARE 2013, 18:1-12.

23. Neill AL, Hunt NH: Pathology of fatal and resolving Plasmodium berghei cerebral malaria in mice. Parasitology 1992, 105:165-175.

24. Huber JD, Campos CR, Mark KS, Davis TP: Alterations in blood-brain barrier ICAM-1 expression and brain microglial activation after lambdacarrageenan-induced inflammatory pain. Am J Physiol Heart Circ Physiol 2006, 290:H732-H740.

25. Shear HL, Shear HL, Marino MW, Wanidworanun C, Berman JW, Nagel RL Correlation of increased expression of intercellular adhesion molecule-1, but not high levels of tumor necrosis factor-alpha, with lethality of Plasmodium yoelii 17XL, a rodent model of cerebral malaria. Am J Trop Med Hyg 1998, 1998(59):852-858.

26. Cserti-Gazdewich CM: Plasmodium falciparum malaria and carbohydrate blood group evolution. ISBT Sci Ser 2010, 5:256-266. doi:10.1111/j.17512824.2010.01380.x.

27. Zhu HT, Bian C, Yuan JC, Chu WH, Xiang X, Chen F, Wang CS, Feng H, Lin $J K$ : Curcumin attenuates acute inflammatory injury by inhibiting the TLR4/MyD88/NF-KB signaling pathway in experimental traumatic brain injury. J Neuroinflammation 2014, 11:59. doi: 10.1186/1742-2094-11-59.

28. Clark DL, Su S, Davidson EA: Saccharide anions as inhibitors of the malaria parasite. Glycoconj J 1997, 14:473-479. 
29. Xiao L, Yang C, Patterson PS, Udhayakumar V, Lal AA: Sulfated polyanions inhibit invasion of erythrocytes by Plasmodium merozoites and cytoadherence of endothelial cells to parasitized erythrocytes. Infect Immun 1996, 64:1373-1378.

30. Recuenco FC, Kobayashi K, Ishiwa A, Enomoto-Rogers Y, Fundador NGV, Sugi T, Takemae H, Iwanaga T, Murakoshi F, Gong H, Inomata A, Horimoto T, Iwata T, Kato K: Gellan sulfate inhibits Plasmodium falciparum growth and invasion of red blood cells in vitro. Sci Rep 2014, 4:4723. doi:10.1038/ srep04723.

doi:10.1186/1475-2875-13-487

Cite this article as: Recuenco et al:: Lambda-carrageenan treatment exacerbates the severity of cerebral malaria caused by Plasmodium berghei ANKA in BALB/c mice. Malaria Journal 2014 13:487.

\section{Submit your next manuscript to BioMed Central} and take full advantage of:

- Convenient online submission

- Thorough peer review

- No space constraints or color figure charges

- Immediate publication on acceptance

- Inclusion in PubMed, CAS, Scopus and Google Scholar

- Research which is freely available for redistribution 